\title{
O Desenvolvimento do Efeito da Outra Raça (EOR) em Crianças: Dos Modelos de Codificação de Faces à Emergência do EOR
}

\author{
The Development of the Other Race Effect (ORE) in Children: From Face \\ Coding Mechanisms to the Emergence of the ORE
}

\author{
Ana Carolina Monnerat Fioravanti-Bastos ${ }^{a}$, Maria Lucia Seidl-de-Moura ${ }^{a}$ \\ \& J. Landeira-Fernandez ${ }^{*}, b, c$ \\ ${ }^{a}$ Universidade do Estado do Rio de Janeiro, Rio de Janeiro, Rio de Janeiro, Brasil, \\ ${ }^{b}$ Pontifícia Universidade Católica do Rio de Janeiro, Rio de Janeiro, Rio de Janeiro, Brasil \\ \& 'Universidade Estácio de Sá, Rio de Janeiro, Rio de Janeiro, Brasil
}

\begin{abstract}
Resumo
Em tarefas de reconhecimento facial, adultos e crianças apresentam dificuldade ao reconhecer faces de raças diferentes da sua. Esse efeito é conhecido como Efeito da Outra Raça (EOR) e tem sido consistentemente replicado em diversos estudos. O objetivo deste trabalho é apresentar uma revisão da literatura sobre alguns modelos teóricos que explicam sua emergência e desenvolvimento. Os modelos de codificação de faces baseado no Protótipo, e em Exemplares são a base para a explicação das diferenças na codificação entre faces da mesma raça e faces de outra raça. Uma revisão das recentes pesquisas sobre o EOR e como experiências com faces de outra raça, durante a primeira infância o influenciam, foi apresentada. Finalmente, pesquisas futuras relativas ao contexto cultural foram propostas, para melhor investigar o desenvolvimento do efeito da raça e dos modelos de codificação de faces.

Palavras-chave: Reconhecimento de faces, efeito da raça, desenvolvimento.
\end{abstract}

\begin{abstract}
In face recognition tasks, adults and children have difficulty recognizing faces from other races. This effect is known as the Other Race Effect (ORE) and has been consistently replicated in several studies. The aim of this paper is to present a review of some theoretical models that explain the ORE, its emergence and development. Face coding mechanisms based on Prototypes and Exemplars are the basis for the explanation of differences in coding between the same race and other race faces. It was presented a review of recent research on ORE and how the experiences with other race faces during early childhood influences the effect. Finally, further research on the cultural context has been proposed to better investigate the development of ORE and face coding mechanisms.
\end{abstract}

Keywords: Face recognition, race effect, development.

Um fenômeno bastante robusto vem sendo identificado e estudado na literatura e consiste no reconhecimento mais preciso de uma face de nossa própria raça do que de uma face de outra raça. A este efeito (Brigham \& Malpass, 1985; Chance \& Goldstein, 1996), dá-se o nome de "Viés da Raça" ou "Efeito da Outra Raça" (EOR). Alguns pesquisadores sugerem que esse efeito pode ser explicado pelo fato do ser humano desenvolver formas especializadas de

\footnotetext{
* Endereço para correspondência: Núcleo de Neuropsicologia Clínica e Experimental, Laboratório de Análise de Dados, Departamento de Psicologia, Pontifícia Universidade Católica do Rio de Janeiro, Rua Marquês de São Vicente, 225, Rio de Janeiro, RJ, Brasil 22453-900. E-mail: landeira@puc-rio.br
}

perceber as características das faces de sua própria raça (Lindsay, Christian, \& Jack, 1991). Outros pesquisadores sugerem que o grau de contato e a experiência com pessoas de outras raças diminuem o EOR, explicando a mitigação deste efeito pelo contato e experiências miscigenadas (Furl, Phillips, \& O'Toole, 2002; Levin 2000).

Para discutir os modelos explicativos para o fenômeno e sua ontogênese é importante a perspectiva da Psicologia Evolucionista (PE), abordagem que propõe estudar a mente humana como resultante de processos adaptativos originados por seleção natural em nosso Ambiente Ancestral de Evolução (AAE; Barkow, Cosmides, \& Tooby, 1992). A PE pressupõe a existência de uma natureza humana universal, constituída de mecanismos psicológicos de processamento de informação que permitem a produção, 
a absorção, a modificação e a transmissão de cultura, adaptadas ao ambiente evolutivo, ou seja, o modo de vida de nossos ancestrais caçadores-coletores do Pleistoceno. Com esta perspectiva podemos pensar o processo de reconhecimento de faces como um desses mecanismos selecionados de processamento de informação.

Seres humanos vivem em ambientes sociais complexos. Esse modo de vida tem vantagens porque oferece segurança e permite ações coletivas, tendo como consequência um conjunto de pressões seletivas sobre os que dele participam. Para sua sobrevivência, foram selecionados mecanismos psicológicos que permitem a compreensão das ações, intenções e emoções de co-específicos. Se um indivíduo pode entender melhor a mente de seus parceiros, tem vantagem sobre os outros, porque consegue cooperar melhor, detectar quem coopera e quem não o faz, se está sendo enganado ou não, e também, às vezes, enganar e manipular outros sem ser descoberto (Kurzban \& Neuberg, 2005).

Somos uma espécie social, de filhotes altriciais, que nascem dependentes de cuidados dos adultos da espécie para sua sobrevivência e, para tal, alguns módulos parecem ter sido selecionados. Sabe-se agora (vide Seidl de Moura \& Ribas, 2004, para uma discussão dessa literatura) que os bebês nascem capazes de discriminar entre estímulos diversos e lhes responder diferencialmente. O primeiro aspectos talvez seja a predisposição a reconhecer e processar estímulos e padrões relacionados a coespecíficos (vozes humanas, de preferência femininas, configurações de faces, cheiro do leite da mãe, língua de seu grupo cultural, contingências, calor emocional etc.). Dentre essas capacidades está a de discriminar configurações de faces humanas e de preferir olhar para elas, a outros tipos de configurações (Kelly et al., 2005). Essa capacidade inicial é a base para o reconhecimento facial que tem importância óbvia para a sobrevivência da nossa espécie, uma vez que esta capacidade permite as trocas entre os sujeitos, o estabelecimento de coalizões etc. (Seeck et al., 2001).

Se o reconhecimento de faces tem origem nesta capacidade de nossa espécie; cabe indagar se o EOR no reconhecimento de faces poderia ter uma explicação filogenética específica. Acreditamos que a resposta é negativa. Um teste simples, para saber se um determinado mecanismo pode ser pensado como parte de nossa mente adaptada, ou seja produto da seleção natural, é pensar se esse mecanismo resolveria problemas que nossos ancestrais encontravam no ambiente ancestral. Neste caso, as evidências sugerem uma resposta negativa. Kurzban, Tooby e Cosmides (2001) propõem que a categorização de raça é um subproduto de adaptações que evoluíram para detectar coalizões e outras alianças de cooperação. Somos capazes de usar pistas de raça para fazer categorizações quando raça é uma pista importante, mas nossa capacidade é para usar pistas relevantes, em geral.

Com essas premissas, de que temos um mecanismo adaptado para reconhecer coespecíficos, que incluiu prefe- rência por configurações de faces e que estamos equipados para usar pistas relevantes para estabelecer categorizações, cabe analisar os mecanismos cognitivos hipotetizados para esse processo. Um dos modelos cognitivos de codificação de faces mais empregados para delinear as causas do EOR, tanto em crianças quanto em adultos, é baseado numa noção de espaço de múltiplas dimensões (Rhodes, Brennan, \& Carey, 1987; Valentine, 1991; Valentine \& Bruce, 1986; Valentine \& Endo, 1992).

De acordo com tal modelo, faces são representadas como vetores num espaço multidimensional. No centro desse espaço, ou seja, na conjunção desses vetores, estaria a média de todas as faces vistas pela pessoa durante seu período de vida. Esta conjunção de vetores é também conhecida como "Norma" ou "Protótipo Facial". A percepção de uma face seria obtido, então, a partir das diferenças entre a face a ser reconhecida e o Protótipo ou Norma (Rhodes et al., 1987; Valentine, 1991).

Com objetivo de delinearmos, melhor, os mecanismos subjacentes ao efeito da raça e o seu desenvolvimento em crianças, apresentaremos uma breve revisão teórica, abordando o Espaço Multidimensional de faces, os Modelos de codificação e o desenvolvimento do Efeito da Outra Raça.

\section{O Espaço Multidimensional de Codificação de Faces}

Uma questão que precede essa discussão é se o reconhecimento de faces pode ser explicado por modelos gerais de categorização, ou se este tem especificidades. Considerando seu valor adaptativo e a presença de mecanismos básicos em recém-nascidos humanos, pode-se hipotetizar que se trata de um módulo ou domínio específico (Barkow et al., 1992; Fodor, 1983). Fodor (1983) propõe que faces são "candidatas favoritas" a formarem um tipo de estímulo que é processado por um módulo específico da mente. Para esse autor, módulos são órgãos mentais especializados, que evoluíram para processar informação específica relevante para a espécie. São faculdades mentais e têm um funcionamento automático, não controlado pelo processador central, estando fora, inclusive, do controle consciente. Com isso, operam com muita rapidez e eficiência.

Outra hipótese seria de que o reconhecimento de faces é tratado por um processador central e que é parte de um processo cognitivo geral, que há muito vem sendo estudado a partir das investigações de um dos fundadores do movimento cognitivista, J. Bruner. Bruner (1983) iniciou o estudo de formação de conceitos e seus ex-estudantes, como E. Rosch (1983) ampliaram e modificaram suas ideias iniciais, abandonando a visão clássica de conceitos e introduzindo a de exemplares ou protótipos. A identificação de exemplares prototípicos seria, para eles, a base para o processo de categorização no caso do desenvolvimento de conceitos naturais, que não poderiam ser criados arbitrariamente pelo experimentador. 
Modelos cognitivos de reconhecimento visual devem especificar como o conhecimento previamente adquirido e armazenado é usado para facilitar o reconhecimento de um estímulo visual. De acordo com Palmier (1975), o reconhecimento de faces é um exemplo de processamento de uma categoria perceptiva que deve incluir uma busca a um conhecimento armazenado na memória. Este conhecimento é armazenado sob a forma de "Valores Prototípicos", ou seja, uma tendência central das dimensões relevantes do estímulo, nesse caso, as faces.

Para Palmier (1975) e outros autores desta perspectiva, ocorre o efeito da distinção no processo de reconhecimento de faces. Isso significa que, a partir da experiência com faces, o sujeito constrói um modelo interno prototípico que é utilizado para facilitar o reconhecimento das faces que essa pessoa encontrará subsequentemente (Bruce, Burton, \& Dench, 1994). As características distintivas de uma face, a orientação sobre a qual ela esta sendo observada, a raça, o sexo, entre outros, são fatores que influenciam na habilidade de um observador em reconhecer essa face. A similaridade ou não entre as faces é gerada pelo efeito desses diversos fatores em conjunto, contrastados ao protótipo armazenado.

Ao pensarmos no espaço de codificação de faces como um espaço de múltiplas dimensões, cada dimensão do espaço representaria características fisionômicas usadas para codificar faces. De fato, o processo envolvido na codificação das faces é baseado em informações frequentes dos valores das características descritivas da face (Valentine, 1991). Entretanto, as dimensões parecem cooperar mais naturalmente com a discriminação dentro de uma classe de estímulos onde todos os exemplares dividem uma estrutura comum, como as faces. Essa visão concorda com a definição de Garner (1978), de uma dimensão como um "atributo que existe para cada estímulo em um conjunto relevante de alguns valores positivos e mutuamente excludentes" (p. 104).

Um estudo com faces caucasianas com sujeitos da mesma raça, que empregou técnicas de escalas multidimensionais, sugere que a principal dimensão necessária no espaço de processamento de faces representa características simples, como cor do cabelo, comprimento, formato do rosto e idade (Shepherd \& Deregowski, 1981). A raça, o gênero e a idade constituem atributos de múltiplas características. Por exemplo, faces femininas e masculinas diferem pela sobrancelha, o tamanho da mandíbula, e etc. (Baudouin \& Tiberghien, 2002). Usamos o formato dos olhos, o tamanho da sobrancelha e da mandíbula para decidirmos se uma face pertence a um homem ou a uma mulher, mas não atribuímos um nariz e boca grandes ou a cor dos olhos como base para essa decisão.

É importante ressaltar que o número de dimensões pode ser grande o suficiente para representar qualquer aspecto da face. Lee, Byatt e Rhodes (2000) utilizaram técnicas de análise fatorial entre as dimensões para calcular a distância que a representação de cada face tem do centro do espaço multidimensional e a densidade de pontos onde ela é alocada. Os resultados demonstraram que a alta correlação positiva entre dois índices corrobora a premissa de que, quanto mais perto a face está do centro, mais densa é a região que ela está alocada. O Espaço Multidimensional onde codificamos faces tem sua origem no centro das dimensões. Sendo assim, podemos dizer que a origem do Espaço Multidimensional é definida como a tendência central das dimensões.

\section{Codificação de Faces: Modelo do Protótipo e Modelo dos Exemplares}

A literatura nos aponta para a existência de dois modelos de codificação de faces. Ambos baseados na premissa de que faces são codificadas como pontos independentes dentro de um espaço de múltiplas dimensões. O modelo da codificação baseado na Norma ou Protótipo pressupõe que faces são codificadas referindo-se a uma Norma captada ou um Protótipo. O modelo baseado em Exemplares assume que as faces são codificadas em função de exemplares categóricos armazenados na memória. Apesar de serem baseados na mesma premissa, o Espaço Multidimensional, os dois modelos fazem predições diferentes.

Segundo a Hipótese do Protótipo, as faces devem ser codificadas em função de um Protótipo, ou Norma, resultante da generalização de todas as faces vistas por uma pessoa durante sua vida (Valentine, 1991). O Protótipo facial seria assim uma imagem ideal armazenada na memória de longo prazo, como a tendência central de uma única classe, representando a aparência típica de apenas um grupo de pessoas.

Neste modelo assumimos que as faces são codificadas em termos do desvio ou distância que elas apresentam em relação a uma que está localizado na origem ou centro do espaço multidimensional, como ilustra a Figura 1A. O reconhecimento de uma face ocorre, então, pela sua comparação com o protótipo através das múltiplas dimensões que formam o estimulo "face" (Valentine, 1991). Se o número de dimensões dentro do espaço facial é denotado por $n$, apenas um vetor " $n$ dimensional" vai partir da norma (origem) em direção ao ponto que representa os valores de dimensões de uma face particular. É este vetor com " $n$ " dimensões que vai unicamente, especificar aquela face. Neste modelo, o reconhecimento de faces é definido como um processo de dois estágios: No primeiro estágio, um estímulo facial é codificado, como um vetor " $n$ dimensional". No segundo estágio, um processo de decisão é requerido para determinar se o estímulo "combina" com um vetor de uma face anteriormente conhecida ou não (Valentine, 1991). 
Fioravanti-Bastos, A. C. M., Seidl-de-Moura M. L. \& Landeira-Fernandez, J. (2014). O Desenvolvimento do Efeito da Outra Raça (EOR) em Crianças: Dos Modelos de Codificação de Faces à Emergência do EOR.

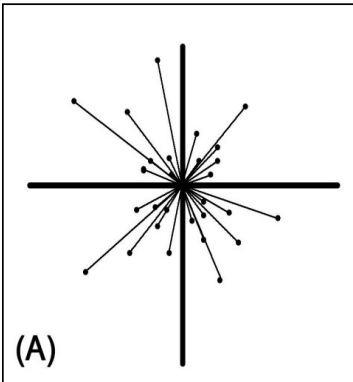

(B)

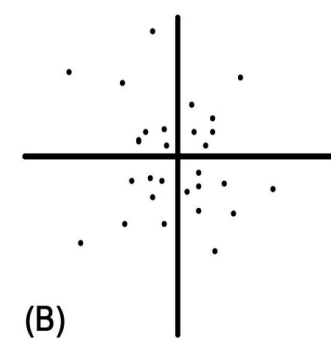

Figura 1. Representações bidimensionais para ilustrar o Modelo do Espaço Multidimensional. (A) é uma representação da codificação Baseado na Norma. Cada ponto representa uma face previamente vista localizado no espaço $n$ dimensional. A origem dos pontos representa o Protótipo. A similaridade entre duas faces é dada pela distância vetorial entre as faces. (B) é uma representação bidimensional ilustrativa do Modelo do Espaço Multidimensional baseado nos Exemplares. Assim como na Figura A, cada ponto representa uma face já vista localizada no espaço $n$ dimensional. A origem dos pontos é arbitrária e representa a o ponto de maior densidade de faces. A similaridade entre as faces é determinada pela distância entre dois pontos e é independente do ponto de origem.

É evidente que todo processo cognitivo de codificação pode envolver algum erro ou ruído e que vai depender das condições do momento da codificação (Valentine \& Bruce, 1986). Esse erro pode ser relativamente grande e é representado como a região de incerteza ao redor das coordenadas do vetor do estímulo. Assumimos então, que a confiança gerada pelo processo de decisão depende de três variáveis: o erro associado ao vetor derivado do estímulo facial; a medida de similaridade entre o vetor derivado do estímulo e o vetor correspondente à face conhecida mais próxima; a similaridade entre o vetor estímulo e o vetor do vizinho mais perto (Valentine, 2001).

Vamos considerar o caso de uma face vista pela primeira vez por um sujeito. Por ser inédita para este indivíduo, não existe nenhuma descrição desta face armazenada na memória. Se a face em questão apresentar características exacerbadas, diferentes daquelas que temos descrições na memória (um nariz muito grande, ou olhos muito afastados, por exemplo) ela será codificada em uma região onde densidade dos pontos ao redor dela será baixa. Entretanto, se a face encontrada possui características mais homogêneas, (ou seja, for uma face típica) sua localização, dentro do espaço de codificação estará em uma região com alta densidade de pontos (Bursey, 1998). Consequentemente, $\mathrm{o}$ reconhecimento de faces distintas será mais rápido $\mathrm{e}$ acurado, uma vez que as faces mais típicas, como estão muito perto uma das outras e em maior quantidade, geram mais "falsos positivos" (Valentine, 1991; Valentine \& Bruce, 1996).

Algumas evidências que suportam o Modelo do Protótipo no reconhecimento de faces vêm de estudos com caricaturas. Rhodes et al. (1987), por exemplo, estudaram o reconhecimento de caricaturas geradas por computadores.
Os autores descrevem como caricatura o processo onde características distintas das faces são exageradas, individualizando, ainda mais, uma face em particular. No estudo, uma face representativa da norma foi gerada pela média das posições das características das faces (nariz, olhos, boca) cruzando várias faces ao mesmo tempo. As caricaturas, por sua vez, foram geradas através do aumento da distância entre a localização de cada característica na face de um individuo e a Norma, seguindo uma proporção fixa. Uma "anticaricatura" também foi gerada através da redução da diferença entre a face e a Norma. Seus resultados demonstraram que caricaturas são reconhecidas mais facilmente do que os desenhos de linhas verídicas, ou desenhos de faces "Normais". Estas, por sua vez, foram reconhecidas mais facilmente do que "anticaricaturas". Os resultados foram interpretados como uma evidência da codificação de faces baseado em protótipos.

Um segundo modelo cognitivo de explicação do reconhecimento de faces é conhecido como modelo de codificação de faces baseado em Exemplares, diferindo do modelo baseado na Norma apenas por partir do princípio de que não existe uma Norma ou Protótipo de onde emergem os vetores de codificação de faces.

De acordo com o modelo dos Exemplares, as faces não são codificadas como vetores dentro do Espaço Multidimensional, mas sim como pontos. A origem desse Espaço Multidimensional não é importante na codificação do estímulo, uma vez que apenas indica o ponto de maior densidade de exemplares, como ilustra a Figura 1B (Valentine, 1991). Esse modelo pressupõe que a similaridade entre duas faces é uma função monotônica da distância que separa as representações de faces no Espaço Multidimensional. O processo de decisão depende de três variáveis: o erro estimado associado à codificação dos estímulos; a distância entre a localização do estímulo e a face conhecida mais próxima; a distância entre o estímulo e seu próximo vizinho.

É importante notar que apesar de ambos os modelos (baseados na norma ou em exemplares) serem modelos de proximidade entre estímulos, eles diferem em relação ao papel de uma norma abstrata de codificação e no uso de uma medida de similaridade de um vetor ou de distância entre pontos (Valentine, 2001).

Ambos os modelos de codificação levam em consideração a distinção que uma face tem de outra em tarefas de reconhecimento. Tal distinção é sempre baseada na quantidade de exemplares de faces presentes no Espaço Multidimensional (Valentine, 2001). Como foi visto, no primeiro modelo, a origem do espaço multidimensional facial é definido como a tendência central das dimensões. Os valores das dimensões que caracterizam as faces vão variar como uma curva Normal ao redor da tendência central. Sendo assim, faces que a pessoa teve mais contato durante a vida estarão mais próximas da tendência central e em maior quantidade. Desta forma, a densidade dos pontos (por exemplo, o numero de faces previamente vistas) vai diminuindo enquanto a distância da tendência 
central vai aumentando; aumentando também o tamanho dos vetores que ligam a face à tendência central. A codificação de uma nova face será alocada em uma dessas duas regiões, de maior ou menor densidade dentro do Espaço Multidimensional (Valentine, 1991; Valentine \& Bruce, 1986; Valentine \& Endo, 1992).

Tanto o modelo baseado na Norma quanto o baseado em Exemplares claramente predizem que o efeito da distinção, ou seja, o quão distinta uma face é da outra, é estudado em termos de densidade de exemplares. A diferença está no fato de que no modelo de codificação a partir da Norma, a similaridade entre duas faces que são equidistantes no espaço é dependente da distância dos pontos em relação à Norma, ou do comprimento do vetor (Valentine \& Bruce, 1986).

\section{O Efeito da Raça no Reconhecimento de Faces}

Como descrito anteriormente, um efeito relativamente bem observado em tarefas de reconhecimento facial é o "Viés da Raça" ou "Efeito da Outra Raça (EOR)". O EOR foi observado inicialmente por Malpass e Kravitz (1969) e corresponde à tendência da pessoa em responder corretamente ao identificar faces de pessoas da mesma raça, em detrimento as faces de raças diferentes da sua.

De fato, é comum acharmos que faces de outras raças parecem mais similares umas com as outras do que faces de nossa própria raça. No inicio do século passado, Feingold (1914) já postulava que para um americano que pouco convive com asiáticos, faces de pessoas desta raça se parecem muito. O inverso também foi constatado. Para asiáticos, todos os caucasianos se parecem. $\mathrm{O}$ efeito do viés da mesma raça tem sido muito replicado, tanto em laboratório quanto em ambientes naturais (Bothwell, Brigham, \& Malpass, 1989; Brigham \& Malpass, 1985; Shepherd \& Deregowski, 1981).

Um paradigma utilizado para analisar o EOR é a tarefa do "atraso no jogo de amostra". Neste paradigma, uma fotografia alvo de uma face de pessoa de raça diferente do participante é rapidamente apresentada, seguida por duas outras fotografias. O participante deve então selecionar aquela que melhor se casa com o alvo. A mesma tarefa é repetida com uma face alvo da mesma raça do participante (Lindsay et al., 1991, Sangrigoli \& de Schonen, 2004). O efeito da raça é evidenciado quando do melhor desempenho do participante em reconhecer faces de sua própria raça.

Diversos estudos apóiam a teoria de que a origem deste efeito está na forma de codificação de estímulos faciais. De acordo com esta visão, a configuração dos estímulos faciais difere de raça para raça, tanto que pessoas desenvolvem conhecimentos especializados ao processar faces de cada raça em particular. Deste modo, por exemplo, sujeitos africanos tendem a direcionar mais atenção para o formato e a posição dos olhos do que para a cor deles (Ellis, Deregowski, \& Shepherd, 1975; Goldstein \& Chance, 1985; Rhodes, Brake, Taylor, \& Tan, 1989).
De acordo com esta perspectiva, Lindsay et al. (1991), sugeriram que as diferenças nas habilidades perceptivas específicas no processamento de faces de uma raça em particular contribuem, de forma significativa, para o Modificações feitas efeito do viés da raça. Comparativamente, indivíduos caucasianos obtiveram melhor desempenho no reconhecimento de suas próprias raças ao passo que os afro-americanos não.

Outros estudos indicaram maior variabilidade do efeito do viés da raça em sujeitos afro-americanos do que em sujeitos caucasianos (Bothwell et al., 1989). Antony, Copper e Mullen (1992), seguindo a mesma linha, encontraram evidências de que o efeito do viés da raça é maior entre sujeitos caucasianos do que afro-americanos. Este padrão também foi encontrado em outros estudos (Cross, Cross, \& Daly, 1971; Malpass \& Kravitz, 1969; Shepard, 1980). Os resultados dessas pesquisas podem ser justificados pela escolha do contexto das amostras. Os dados foram coletados em contextos culturais com maioria caucasiana e minoria afro-americana (em cidades dos Estados Unidos). Sendo assim, os afro-americanos têm contato com maior numero de faces caucasianas e menor numero de faces negras, em comparação com os caucasianos. Cabe compreender se essa diferença nas habilidades de reconhecer faces ocorre por conta do contato com pessoas de outra raça, ou por conta das diferenças nas características faciais entre as raças. Brigham e Malpass (1985) encontraram algumas evidências diretas de que o viés da raça é produto de diferentes tipos de interações entre membros de diferentes raças na sociedade.

Três correntes teóricas que buscam explicar o EOR em função das características da face e do contato com faces de outra raça. São elas: a Hipótese das Diferenças Inerente aos Estímulos, proposta por Chance e Goldenstein (1996); a Hipótese da Atitude Social, proposta por Seeleman (1940); a Hipótese da Experiência Diferencial, proposta por Cross et al. (1971).

A primeira hipótese propõe que algumas raças possuem características faciais mais homogêneas que outras. Autores que defendem esta hipótese são Herrera e seus colaboradores (Herrera, McQuistonet, McLin, \& Malpass, 2000) e sugerem que o efeito do viés da raça não é necessariamente causado pela falta de experiência com outras raças, mas sim atribuído a caracterizações perceptivas da raça. Esses autores examinaram o efeito do viés da raça com participantes hispânicos, usando como estímulo faces racialmente ambíguas. As faces ambíguas foram criadas de modo a que as características faciais (olho, boca e nariz) se sobrepusessem às linhas faciais de hispânicos e negros. O cabelo foi usado como uma marca, sem ele a determinação da raça se torna mais difícil. Observou-se que participantes hispânicos reconheceram melhor faces hispânicas do que negras. Isso pode ser atribuído a maior porcentagem de falsos alarmes para faces negras e a porcentagem de acertos iguais para faces negras e hispânicas. Os resultados não apresentaram diferença estatística na habilidade 
Fioravanti-Bastos, A. C. M., Seidl-de-Moura M. L. \& Landeira-Fernandez, J. (2014). O Desenvolvimento do Efeito da Outra Raça (EOR) em Crianças: Dos Modelos de Codificação de Faces à Emergência do EOR.

de reconhecer faces "velhas" (faces bastante conhecidas) caucasianas ou hispânicas. Entretanto, faces "novas" (que eles acabaram de conhecer) negras foram mais falsamente reconhecidas do que faces "novas" hispânicas, o que leva a concluir que hispânicos são mais acurados em reconhecer corretamente faces hispânicas não vistas anteriormente. Esses resultados apóiam parcialmente a hipótese de diferenças entre as faces de diferentes raças.

A segunda hipótese, geralmente oferecida como explicação para o efeito da raça, é a Hipótese da Atitude Social, que propõe que as atitudes interraciais dos indivíduos afetam suas habilidades de reconhecimento facial. Galper (1973) buscou testar essa hipótese avaliando estudantes americanos caucasianos, que frequentavam cursos com afro-americanos. Seus resultados indicaram que aqueles que tinham desenvolvido relações de amizade mais próximas aos estudantes afro-americanos eram capazes de reconhecer faces negras com mais precisão do que aqueles estudantes que, mesmo frequentando contextos miscigenados, não desenvolveram relações de amizades entre raças. Feinman e Entwistle (1976) também obtiveram resultados na mesma direção com crianças americanas que têm amigos de outras raças. Os pesquisadores demonstraram, em um estudo com 288 crianças (caucasianas e negras), que aquelas crianças que estudam em escolas integradas (com alunos caucasianos e negros) e moram em bairros miscigenados, desenvolvendo relações de amizade entre raças, exibem menos o efeito do viés da raça do as crianças que vivem em bairros e estudam em escolas segregadas, não tendo relações entre as raças.

A terceira hipótese, da Experiência Diferencial, faz predições muito parecidas com a hipótese anterior. Ela propõe que o nível de experiência e contato com pessoas de outras raças afeta a habilidade de reconhecimento de faces. Cross et al. (1971) observaram que pessoas caucasianas que vivem em comunidades com grande incidência de negros e caucasianos exibiram maior habilidade em reconhecer faces negras do que as pessoas caucasianas que vivem em comunidades segregadas.

Apoiando ainda essa hipótese, outros estudos mais recentes têm demonstrado que o efeito da raça diminui de acordo com o aumento da experiência com faces de outras raças (de Heering, de Liedekerke, Deboni, \& Rossion 2010; Sangrigoli \& de Schonen, 2004), ou que o tempo de exposição com faces de outra raça pode reduzir ou até mesmo inverter o EOR. Com objetivo de avaliar se o efeito da raça sofre modificações em função da experiência com faces de outra raça, Sangrigoli, Pallier, Argenti, Ventureyra e de Schonen (2005) compararam as habilidades de reconhecer faces de adultos de origem coreana, que foram adotados entre três e 9 anos de idade por famílias européias com um grupo controle de caucasianos europeus. Em seus resultados não foram encontradas diferenças entre os dois grupos para reconhecer os dois tipos de faces (caucasianas e coreanas), demonstrando que a experiência de longos anos com faces caucasianas, foi suficiente para os adultos, de origem coreanas, desenvolverem habilidades superiores para reconhecer faces caucasianas. Este padrão indicou uma reversão do EOR.

Ambas as Hipóteses da Atitude Social e Experiência Diferencial fazem predições muito parecidas, no que diz respeito ao aumento do contato com outra raça ser um fator que influencia a magnitude do EOR, no entanto pesquisadores que apóiam a hipótese da Hipótese da Atitude Social atribuem um peso maior para as escolhas sociais do individuo, de amizades e relacionamentos e pesquisadores que apóiam a Hipótese da Experiência Diferencial dizem que apenas o contexto em que a pessoa vive - comunidade segregada ou não é importante para modular a magnitude do EOR. A diferença entre as duas correntes teóricas pode ser explicada em função da importância do envolvimento emocional inerente as escolhas de amizade e relacionamento pessoal, podendo ajudar na precisão do reconhecimento de faces de outras raças.

Paralelamente às três Hipóteses descritas, alguns estudos demonstram como faces de outra raça são codificadas dentro do espaço multidimensional. Quando se trata de reconhecer faces alheias à raça do observador, este teria a dificuldade de assimilar os elementos peculiares e particulares presentes. Isto ocorreria por não possuir experiência suficiente com uma determinada população para se identificar o que é característica peculiar em apenas uma das faces (Valentine \& Endo, 1992).

De acordo com o modelo Baseado na Norma ou Protótipo, Modificação feita o espaço representacional para faces da mesma raça inclui uma melhor diferenciação das dimensões da face. Como resultado, faces da mesma raça são mais homogeneamente espalhadas pelo Espaço Multidimensional e representadas de forma mais distinta, sendo reconhecidas com mais precisão. $\mathrm{O}$ pouco contato que a pessoa teve com faces de outra raça gera um Protótipo inacabado, ou inexistente, para as características dessas faces. Aumentando o contato com uma raça diferente, passa-se a identificar melhor a face. A explicação do modelo baseado no Protótipo para esse fato, é que o contato com as faces de outra raça aumenta a versatilidade do Protótipo estendendo-o e tornando-o aplicável à nova raça.

Faces de indivíduos de outras raças formam uma classe de estímulos que violam as estatísticas da população de faces da mesma raça, agrupando-se em uma região afastada do Protótipo ou Norma (Valentine \& Bruce, 1986). Ao utilizar técnicas de análise fatorial entre as dimensões de faces de outra raça, para calcular a distancia que cada face tem do centro do espaço multidimensional e a densidade de pontos onde ela é alocada, Caldara e Abdi (2006) não encontraram correlação positiva entre a distância do centro e a densidade da região em que a face está alocada, sugerindo que a codificação de faces de outra raça de fato se comporta de forma diferente no espaço multidimensional, como ilustra a Figura 2. Assim, parece provável que o efeito da raça resulte das diferenças na representação mental entre faces da mesma raça e faces de outra raça. 


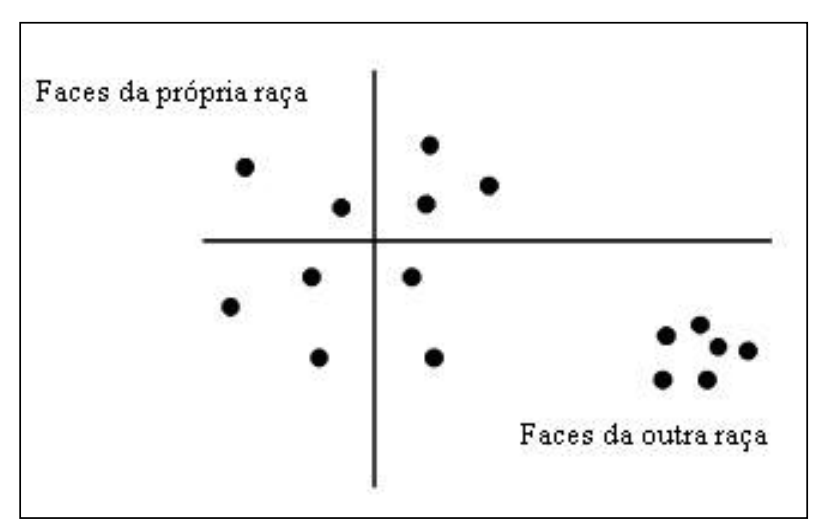

Figura 2. Representação esquemática para faces da mesma raça e faces de outra raça dentro do Espaço Multidimensional. A Figura 2 representa um esquema bidimensional ilustrativo, quando na realidade o espaço é formado por múltiplas dimensões.

Quando se considera o modelo baseado em Exemplares, as diferenças na densidade de exemplares entre faces da própria raça e faces de outra raça podem explicar o EOR (Valentine, 1991; Valentine \& Endo, 1992). As dimensões perceptivas que formam a base do espaço face estão "sintonizadas" para captar a sutil diferença entre as faces. Como a experiência das pessoas tende a ser maior com faces da sua própria raça, as características das dimensões que permeiam o espaço facial são otimizadas para discriminação de faces da própria raça do sujeito.

Nossas habilidades perceptivas com faces de nossa própria raça dão origem a recursos perceptivos que não são generalizáveis para faces de outra raça (Chiroro \& Valentine, 1995; Valentine, 1991; Valentine \& Endo, 1992). Assim, o Modelo baseado em Exemplares também propõe que o efeito da outra raça é resultado da falta de experiência com faces de outra raça (Byatt \& Rhodes, 2004).

Apesar das três hipóteses explicativas do EOR, descritas anteriormente (Diferença Inerente ao Estímulo, Atitude Social, Experiência Diferencial), MacLin e Malpass (2001), demonstraram a importância do fator "raça" que é explicitamente levado em conta quando codificamos faces de outra raça. Por exemplo, ao sermos apresentados a uma fotografia de uma face de outra raça, o primeiro pensamento é: "Essa pessoa é Oriental, ou Negra, ou Caucasiana".

Algumas características que individualizam as faces de outra raça são extraídas e armazenadas em um subgrupo de dimensões afastado do centro do espaço multidimensional, sendo assim, elas não são suficientes para um reconhecimento acurado dessa face. Dimensões relativas a características da raça vão então, receber um peso relativamente maior para faces de outras raças do que para faces da mesma raça do sujeito. Desta forma, há relativamente menos informações de individualização para faces de outra raça. Em contraste, faces da própria raça são essencialmente codificadas em dimensões não relacionadas à raça, mas a características de individualização (MacLin \& Malpass, 2001). Por isso, faces de outra raça e faces da própria raça formam grupos distintos no espaço facial, devido às diferenças relativas à densidade de exemplares entre estes dois tipos de face, como ilustra a Figura 2. De certa forma, os achados de MacLin e Malpass (2001) corroboram a Hipótese das diferenças Inerentes ao Estímulo e caminham no sentido de explicar a codificação destas dentro do espaço multidimensional.

\section{O Efeito da Raça em Crianças}

Além das explicações sobre o processo em adultos, o entendimento de sua ontogênese ainda suscita controvérsias, principalmente quando à idade em que esse efeito começa a ser observado. Alguns resultados são ainda sem explicações teóricas razoáveis. A primeira questão é quão cedo esse efeito pode ser observado. Achados recentes em relação à preferências espontâneas, têm confirmado a influência que diferentes experiências com faces apresentam na forma que o Protótipo adquire durante a primeira infância (Bar-Hain, Ziv, Lamy, \& Hodes, 2006; Kelly et al., 2005; Kelly, Quinn, et al., 2007)

Bar-Hain et al. (2006) testaram crianças da Etiópia criadas em centros de absorção enquanto seus pais tentavam abrigo em Israel. Essas crianças eram frequentemente expostas a adultos etíopes e israelenses e não demonstraram preferências por faces africanas ou caucasianas quando apresentadas simultaneamente. Esses resultados fornecem evidências de que faces observadas no ambiente visual desempenham um importante papel nas preferências faciais suscitadas durante a infância (Quinn, Yahr, Kuhn, Slater, \& Pascalis, 2002).

Sangrigoli e de Shonen (2004) demonstraram que crianças caucasianas de três meses de idade são capazes de reconhecer faces de sua própria raça, mas não faces de asiáticos. Entretanto, o efeito desapareceu quando a criança era habituada a três, e não apenas um exemplar da face de outra raça. Seus resultados demonstram que, mesmo que o efeito da raça esteja presente em crianças de três meses de idade, ele é fraco o suficiente para ser eliminado após alguns instantes de exposição dentro de uma sessão experimental.

A seletividade baseada nas diferenças faciais raciais parece emergir muito cedo na vida. Com três meses de idade, crianças preferem olhar para faces de seu grupo do que para faces de outro grupo racial (Kelly, Ge, et al., 2007). Entretanto essa preferência não está presente no nascimento, o que sugere fortemente que preferências por seus próprios grupos resultam de diferentes exposições a faces do grupo racial de cada pessoa (Kelly et al., 2005).

Kelly, Quinn, e colaboradores (2007) investigaram a latência do EOR durante os primeiros meses de vida. Seus resultados não demonstraram evidências do efeito do viés da raça em crianças de três meses. Nesses estudos observou-se que o reconhecimento de faces de sua própria raça, emerge aos seis meses e está totalmente presente aos nove meses de idade. Seus resultados demonstraram que Efeito da Raça segue alguns passos de desenvolvimento. Primeiramente, a exposição a faces do mesmo grupo racial 
Fioravanti-Bastos, A. C. M., Seidl-de-Moura M. L. \& Landeira-Fernandez, J. (2014). O Desenvolvimento do Efeito da Outra Raça (EOR) em Crianças: Dos Modelos de Codificação de Faces à Emergência do EOR.

da criança recém nascida induz a uma familiaridade e a preferências visuais por tais faces. Em um segundo momento, as preferências pelas faces de seu mesmo grupo racial produzem maior atenção visual para essa face, mesmo quando faces de outros grupos raciais estão presentes no mesmo ambiente (Bar-Hain et al., 2006; Kelly et al., 2005; Kelly, Ge, et al., 2007). Em um terceiro momento, são desenvolvidas habilidades superiores de reconhecimento para faces do mesmo grupo racial da criança, e não para faces de grupos raciais com os quais a criança não é deparada com frequência (Kelly, Quinn, et al., 2007).

Sangrigoli e de Schonen (2004), investigaram as habilidades no reconhecimento facial de crianças caucasianas de três a cinco anos. Os estímulos eram fotografias de faces de adultos asiáticos e caucasianos. Seus resultados indicaram que crianças caucasianas apresentaram um melhor desempenho com relação ao reconhecimento de faces caucasianas. Crianças asiáticas foram melhores nas faces asiáticas. A vantagem das faces de "mesma raça" foi estável para essa faixa de idade, sugerindo que o efeito da outra raça já esta presente em crianças de três anos.

Vários estudos apresentam evidências de que a memória de reconhecimento facial melhora significativamente com a idade. Feinman e Entwisle (1976), por exemplo, testaram crianças americanas, caucasianas e negras nas séries um, dois, três e seis do ensino fundamental nas suas habilidades em reconhecer fotos de crianças caucasianas e negras. O efeito da raça foi evidente. Constatou-se que crianças caucasianas desempenharam melhor a tarefa de reconhecer faces caucasianas do que negras e as crianças negras foram melhores ao reconhecer faces negras do que caucasianas, em todas as faixas de idade.

Pezdek, Blandon-Gitlin e Moore (2003) demonstraram que a memória de reconhecimento facial melhora com a idade e que o Efeito da Raça é evidente tanto em crianças como em adulto. Em seu estudo foi comparado o desempenho de 186 crianças, incluindo 62 em jardim de infância (media de idade 5,63), 62 da terceira série (média de idade 8,63 ) e 62 jovens adultos (média de idade 24,61). Metade das crianças eram negras e a outra metade caucasianas residentes na Califórnia. O delineamento experimental de Pezdek et al. (2003) teve como objetivo aproximar o máximo da situação de testemunha ocular, onde a criança assiste a um crime e depois deve reconhecer o criminoso. Os participantes assistiam a vídeos onde duas pessoas de raças diferentes (negras e caucasianas) desempenhavam uma tarefa de cozinha e retornavam no dia seguinte para identificar as pessoas. Este procedimento difere dos demais estudos, pois utiliza situações mais reais e pessoas desempenhando atividades, e não apenas o jogo de ver uma fotografia e reconhecer a face dentre outras. $\mathrm{O}$ espaço de tempo de um dia para o outro também reforça o desempenho no reconhecimento. Seus resultados indicaram que o efeito da raça está presente em crianças mais novas é o mesmo a partir dos cinco anos de idade. Não apenas faces da própria raça foram mais frequentemente reconhecidas em todos os níveis de idade, como a dimensão do efeito da raça não variou com a idade.

Os estudos citados apóiam tanto a Hipótese da Experiência Diferenciada, quanto a Hipótese da Atitude Social, quando também com crianças com maior contato com faces de outras raças, o efeito da raça tende a diminuir (Chiroro \& Valentine, 1995; Feinman \& Entwisle, 1976). Vários aspectos do processamento facial melhoram com a idade entre 12-14 anos (Bruce et al., 2000; Carey, Diamond, \& Woods, 1980; Mondloch, Le Grand, \& Maurer, 2002). A existência precoce do efeito do viés da raça junto com o desenvolvimento prolongado do processamento facial demonstra que o sistema do processamento facial é desenvolvido parcialmente através da interação com o ambiente em que a criança se encontra.

Sangrigoli e de Shonen (2004) propõem que a plasticidade dos processos cognitivos responsáveis pelo reconhecimento de faces, em especial ao reconhecer faces com características raciais diferentes das do próprio sujeito, ainda está presente até os nove anos e que os processos de identificação podem ser profundamente modificados durante a infância. A idade do início do contato com outras raças deve ser um importante fator para determinar as habilidades de reconhecimento facial de outra raça. De acordo com Sangrigoli e de Shonen (2004), a infância deve ser um período de muita sensibilidade, além do qual, o efeito na experiência de reconhecimento facial deve ser marcadamente reduzido.

Em síntese, os bebês nascem com algumas características que parecem parte de um módulo de reconhecimento de coespecíficos que agem como programas abertos para que aprendam sobre faces. Nessa aprendizagem, suas predisposições transformam-se pela experiência e pelas interações em seus contextos de desenvolvimento.

Um modelo teórico que dá conta dessas transformações e pode explicar o efeito de raça no reconhecimento de faces é o de Karmiloff-Smith (1995, 2000). Essa autora argumenta que a mente humana inclui predisposições inatamente especificadas de natureza domínio-específicas, que estabelecem limites quanto aos tipos de input a serem processados. Essas predisposições atuam na seleção de inputs relevantes pelos bebês humanos. Essas predisposições inatas são epigenéticas a partir dos limites impostos por elas, o desenvolvimento se dá por um processo que a autora denomina redescrição representacional. As informações inatas e adquiridas são transformadas de forma iterativa por sua reapresentação interna em diferentes formatos. Além disso, as redescrições representacionais transformam informação implícita em conhecimento explícito, e observam-se a construção consciente e exploração de analogias, experimentos mentais e reais. O processo é cíclico, acontece recorrentemente dentro de microdomínios (como o de reconhecimento de faces) ao longo do desenvolvimento, e apresenta-se em fases.

Na primeira fase a aprendizagem é orientada pelos dados e o que é focalizado é, predominantemente, in- 
formação do ambiente externo. Com isso, são criadas adjunções representacionais. Essas adjunções somam-se a representações já estáveis, mas não as transformam. $\mathrm{O}$ produto dessa fase é desempenho bem-sucedido em qualquer microdomínio que chegou naquele nível. É o domínio comportamental. Na segunda fase, internamente movida, o foco de mudança é o estado atual das representações de conhecimento em um determinado microdomínio. Adjunções representacionais podem ser transformadas. Finalmente, na terceira fase, chega-se a um equilíbrio entre controle interno e externo, com a integração das representações já construídas e as novas informações recebidas. Adjunções diversas e representações podem interagir, e, transformadas, levam a novas representações de maior grau de complexidade e estabilidade. A transformação do implícito em explícito envolve para Karmiloff-Smith (1995) a "apropriação de estados estáveis". A informação que esses estados contêm é extraída e pode então ser usada de forma mais flexível para outras finalidades.

\section{Considerações Finais}

Com objetivo de apresentar uma revisão teórica a respeito do desenvolvimento do EOR, recorremos a premissa da PE, propondo que nossas preferências por configurações faciais fazem parte de um mecanismo adaptado para reconhecer coespecíficos. Tal mecanismo incluiu preferência por configurações de faces, o que resulta em uma habilidade especifica para usar pistas relevantes para estabelecer categorizações. Esse processo é constituído de mecanismos cognitivos específicos e tem na teoria do Protótipo sua grande base.

$\mathrm{O}$ reconhecimento de uma face ocorre em função da comparação da face vista e um protótipo de faces existente na memória de longo prazo. Essa comparação ocorre em um Espaço Multidimensional através de múltiplos vetores, que determinam as características de cada face. O protótipo começa a se formar desde o nascimento e o sistema de processamento de faces torna-se gradativamente "sintonizado" para processar a categoria de faces mais prevalentes no ambiente visual do bebê, gerando o Efeito da Raça, ou melhor precisão ao reconhecer faces da própria Raça.

De fato, o Efeito da Raça está presente desde muito cedo na primeira infância. A magnitude do efeito da raça tende a ser proporcional ao contato que a criança, e posteriormente o adulto tem com faces de outra raça, expandindo o protótipo ou a quantidade de exemplares com características faciais de diferentes raças (Meissner \& Brigham, 2001). Os Modelos de codificação baseados na Norma e em Exemplares são eficientes explicações para as diferenças no momento da codificação de faces da mesma raça e faces de outra raça.

Experiências com diferentes grupos raciais sintonizam o Espaço Multidimensional de codificação de faces em função das faces destes grupos. Entretanto, pesquisas futuras devem ser propostas no sentido de delinear melhor as diferenças entre crianças e adultos na codificação de faces dentro do Espaço Muldimensional. Precisamos investigar como a plasticidade do protótipo na infância interfere na densidade de exemplares e na distância vetorial entre a face e Norma e ainda compreender como o protótipo se expande para inserir as faces de outra raça, durante a experiência com essas faces na infância.

Outro ponto fundamental para futuras investigações é a questão da universalização do Efeito da Raça. Sabemos que ele surge no início do desenvolvimento, mas não existem dados disponíveis para nos informar as diferenças do desenvolvimento do efeito em diferentes grupos raciais. Uma literatura recente, e ainda em expansão, começa a desafiar a universalidade dos aspectos mais fundamentais da percepção humana. Tem sido demonstrado que diferenças culturais de percepção também existem dentro do domínio de processamento de faces (Blais, Jack, Scheepers, Fiset, \& Caldara, 2008), caracterizando modos profundamente divergentes para extrair e processar a informação visual. Pesquisas futuras devem estender os achados para outras amostras raciais antes de considerar qualquer noção de universalidade do desenvolvimento.

É possível que as crianças criadas em ambientes específicos (por exemplo, birraciais) desenvolvam habilidades diferenciadas no processo de reconhecimento de faces (Bar-Hain et al, 2006; Sugita, 2008). Um desafio para o futuro seria investigar que aspectos do processamento de faces em crianças estão sujeitos às influências culturais e o quão determinantes são essas influências na ontogênese do Efeito da Raça.

\section{Referências}

Antony, T., Copper, C., \& Mullen, B. (1992). Cross-racial facial identification: A social cognitive integration. Personality and Social Psychology Bulletin, 18, 296-301.

Bar-Hain, Y., Ziv, T., Lamy, D., \& Hodes, R. M. (2006). Nature and nurture in own-race face processing. Psychological Science, 17, 159-163.

Barkow, J., Cosmides, L., \& Tooby, J. (Eds.). (1992). The adapted mind: Evolutionary psychology and the generation of culture. New York: Oxford University Press.

Baudouin, J. Y., \& Tiberghien, G. (2002). Gender is a dimension of face recognition. Journal of Experimental Psychology: Learning, Memory, and Cognition, 28, 362-365.

Blais, C., Jack, R. E., Scheepers, C., Fiset, D., \& Caldara, R. (2008). Culture shapes how we look at faces. PLOS ONE 3, e3022.

Bothwell, R. K., Brigham, J. C., \& Malpass, R. S. (1989). Crossracial identifications. Personality and Psychology Bulletin, $15,19-25$.

Brigham, J. C., \& Malpass, R. S. (1985). The role of experience and contact in the recognition of faces of own - And otherraces persons. Journal of Social Issues, 41, 139-155.

Bruce, V., Burton, M. A., \& Dench, N. (1994). What's distinctive about a distinctive face? Quarterly Journal of Experimental Psychology, 47(A), 119-141.

Bruce, V., Campbell, R. D., Doferty-Sneddon, G., Import, A., Langston, S., McAuley, S., \& Wright, R. (2000). Testing face processing skills in children. British Journal of Developmental Psychology, 7, 3-15. 
Fioravanti-Bastos, A. C. M., Seidl-de-Moura M. L. \& Landeira-Fernandez, J. (2014). O Desenvolvimento do Efeito da Outra Raça (EOR) em Crianças: Dos Modelos de Codificação de Faces à Emergência do EOR.

Bruner, J. (1983). In search of mind: Essays in autobiography. New York: Harper \& Row.

Bursey, T. (1998). Physical and psychological representations of faces: Evidence from Morphing. Psychological Science, 9, 476-483.

Byatt, G., \& Rhodes, G. (2004). Identification of own-race and other-race faces: Implications for the representation of race in face space. Psychonomic Bulletin \& Review, 11, 735-741.

Caldara, R., \& Abdi, H. (2006). Simulating the 'other-race' effect with autoassociative neural networks: Further evidence in favor of the face-space model. Perception, 35, 659-670.

Carey, S., Diamond, R., \& Woods, B. (1980). Development of face recognition: A maturational component? Developmental Psychology, 16, 257-269.

Chance, J. E., \& Goldstein, A. G. (1996). The other race effect and eyewitness identification. In S. L. Sporer, R. S. Malpass, \& G. Koehnken (Eds.), Psychological Issues in Eyewitness Identification (pp. 153-176). Mahwah, NJ: Erlbaum.

Chiroro, P., \& Valentine, T. (1995). An investigation of the contact hypothesis of the own-race bias in face recognition. Quarterly Journal of Experimental Psychology: Human Experimental Psychology, 48(A), 879-894.

Cosmides, L., \& Tooby, J. (1992). Cognitive adaptations for social exchange. In J. Barkow, L. Cosmides, \& J. Tooby (Eds.), The adapted mind: Evolutionary psychology and the generation of culture. New York: Oxford University Press.

Cross, J. F., Cross, J., \& Daly, J. (1971). Sex, race, age, and beauty as factors in recognition of faces. Perception and Psychophysics, 10(6), 393-396.

de Heering, A., de Liedekerke, C., Deboni, M., \& Rossion, B. (2010). The role of experience during childhood in shaping the other-race effect. Developmental Science, 13, 181-187.

Ellis, H. D., Deregowski, J. B., \& Shepherd, J. W. (1975). Description of white and black faces by white and black subjects. International Journal of Psychology, 10, 119-123.

Feingold, C. A. (1914). The influence of environment on identification of persons and things. Journal of Criminal Law and Police Science, 5, 39-51.

Feinman, S., \& Entwistle, D. R. (1976). Children's ability to recognize other children's faces. Child Development, 47, 506-570.

Fodor, J. (1983). The modularity of mind. Cambridge, MA: Massachusetts Institute of Technology Press.

Furl, N., Phillips, P. J., \& O'Toole, A. J. (2002). Face recognition algorithms and the other race effect: Computational mechanisms for a developmental contact hypothesis. Cognitive Science, 26, 797-815.

Galper, G. E. (1973). "Functional race membership" and recognition of faces. Perceptual and Motor Skills, 37, 455-462.

Garner, W. R. (1978). Aspects of a stimulus: Features, dimensions, and configurations. In E. H. Rosch \& B. B. Lloyd (Eds.), Cognition and categorization (pp. 99-133). Hillsdale, NJ: Laurence Erlbaum.

Goldstein, A. G., \& Chance, J. E. (1985). Effects of training on Japanese faces recognition: Reduction of the other-race effect. Bulletin of the Psychonomic Society, 23, 211-214.

Herrera, V., McQuiston, D. E., MacLin, O. H., \& Malpass, R. S. (2000). Examining the cross race effect using racially ambiguous Faces. El Paso, TX: University of Texas.

Karmiloff-Smith, A. (1995). Beyond modularity: A developmental perspective on cognitive science. Cambridge, MA Massachusetts Institute of Technology Press.
Karmiloff-Smith, A. (2000). The connectionist infant: Would Piaget turn in his grave? In A. Slater \& D. Muir (Eds.), The Blackwell reader in developmental psychology (pp. 43-52). Oxford, UK: Blackwell.

Kelly, D. J., Ge, L., Liu, S., Quinn, P. C., Slater, A. M., \& Lee, K. (2007). Cross-race preferences for same-race faces extend beyond the African versus Caucasian contrast in 3-month-old infants. Infancy, 11, 87-95.

Kelly, D. J., Quinn, P. C., Slater, A. M., Lee, K., Ge, L., \& Pascalis, O. (2007). The other-race effect develops during infancy: Evidence of perceptual narrowing. Psychological Science 18, 1084-1089.

Kelly, D. J., Quinn, P. C., Slater, A. M., Lee, K., Gibson, A., Smith, M., Ge, L., \& Pascalis, O. (2005). Three-month-olds, but not newborns, prefer own-race faces. Developmental Science, 8, 31-36.

Kurzban, R., \& Neuberg, S. (2005). Managing ingroup and outgroup relationships. In D. M. Buss (Ed.), The handbook of evolutionary psychology (pp. 653-675). Hoboken, NJ: Wiley.

Kurzban, R., Tooby, J., \& Cosmides, L. (2001). Can race be erased? Coalitional computation and social categorization. Proceedings of the National Academy of Sciences, 98(26), 15387-15392.

Lee, K. J., Byatt, G., \& Rhodes, G. (2000). Caricature effects, distinctiveness, and identification: Testing the face-space framework. Psychological Science, 11, 379-385.

Levin, D. T. (2000). Race as a visual feature: Using visual search and perceptual discrimination tasks to understand face categories and the cross-race recognition deficit. Journal of Experimental Psychology: General, 129(4), 559-574.

Lindsay, D. S., Christian, M. A., \& Jack, P. C. (1991). Other-race face perception. Journal of Applied Psychology, 76, 587-589.

MacLin, O. H., \& Malpass, R. S. (2001). Racial categorization of faces: The ambiguous race face effect. Psychology, Public Policy, and Law, 7, 98-118.

Malpass, R. S., \& Kravitz, J. (1969). Recognition for faces of own and other 'race'. Journal of Personality and Social Psychology, 13, 330-334.

Meissner, C. A., \& Brigham, J. C. (2001). Thirty years of investigating the own-race bias in memory for faces: A meta-analytic review. Psychology, Public Policy, and Law, 7, 3-35.

Mondloch, C. J., Le Grand, R., \& Maurer, D. (2002). Configural face processing develops more slowly than featural face processing. Perception, 31, 553-566.

Palmier, R. (1975). Ethnicity, party and national integration. Modern Asian Studies, 9, 130-135.

Pezdek, K., Blandon-Gitlin, I., \& Moore, C. (2003). Children's face recognition memory: More evidence for the cross-race effect. Journal of Applied Psychology, 88(4), 760-763.

Quinn, P. C., Yahr, J., Kuhn, A., Slater, A. M., \& Pascalis, O. (2002). Representation of the gender of human faces by infants: A preference for female. Perception, 31, 1109-1121.

Rhodes, G., Brake, S., Taylor, K., \& Tan, S. (1989). Expertise and configural coding in face recognition. British Journal of Psychology, 80, 313-331.

Rhodes, G., Brennan, S., \& Carey, S. (1987). Identification and ratings of caricatures: Implications for mental representations of faces. Cognitive Psychology, 19, 473-497.

Rosch, E. (1983). Prototype classification and logical classification: The two systems. In E. Scholnick (Ed.), New Trends in Cognitive Representation: Challenges to Piaget's Theory (pp. 73-86). Hillsdale, NJ: Lawrence Erlbaum. 
Sangrigoli, S., \& de Schonen, S. (2004). Effect of visual experience on face processing: A developmental study of inversion and non-native effects. Developmental Science, 1, 74-87.

Sangrigoli, S., Pallier, C., Argenti, A. M., Ventureyra, V. A. G., \& de Schonen, S. (2005). Reversibility of the other-race effect in face recognition during childhood. Psychological Science, 16, 440-444.

Seeck, M., Michel, C. M., Blanke, O., Thut, G., Landis, T., \& Schomer, D. L. (2001). Intracranial neurophysiological correlates related to the processing of faces. Epilepsy and Behavior, 2(6), 545-557.

Seeleman, V. (1940). The influence of attitude upon the remembering of pictorial material. Archives of Psychology, 36(258), 6-69.

Seidl de Moura, M. L., \& Ribas, A. F. P. (2004). Evidências sobre características de bebês recém-nascidos: Um convite a reflexões teóricas. In M. L. S. D. Moura (Ed.), O bebê do século XXI e a psicologia em desenvolvimento (pp. 21-60). São Paulo, SP: Casa do Psicólogo.

Shepard, R. N. (1980). Multidimensional scaling, tree-fitting, and clustering. Science, 210, 390-398.

Shepherd, J. W., \& Deregowski, J. B. (1981). Races and faces - A comparison of the responses of Africans and Whites to faces of the same and different races. British Journal of Social Psychology, 20, 125-133.

Sugita, Y. (2008). Face perception in monkeys reared with no exposure to faces. Proceedings of the National Academy of Sciences of the United States of America, 105(1), 394-398.

Valentine, T. (1991). A unified account of the effects of distinctiveness, inversion, and race on face recognition. Quarterly Journal of Experimental Psychology, 43(2), 161-204.

Valentine, T. (2001). Face-space models of face recognition. In M. J. Wenger \& J. T. Townsend (Eds.), Computational, geometric, and process perspectives on facial cognition: Contexts and challenges (pp. 83-113). Hillsdale, NJ: Erlbaum

Valentine, T., \& Bruce, V. (1986). The effect of race, inversion, and encoding activity upon face recognition. Acta Psychologica, 61, 259-273.

Valentine, T., \& Endo, M. (1992). Towards an exemplar model of faces processing: The effects of race and distinctiveness. Quarterly Journal of Experimental Psychology, 44(4), 671703. 